\title{
Toxicokinetic-toxicodynamic modelling of survival of Gammarus pulex in multiple pulse exposures to propiconazole: model assumptions, calibration data requirements and predictive power
}

\author{
Anna-Maija Nyman • Kristin Schirmer • \\ Roman Ashauer
}

Accepted: 12 April 2012/Published online: 5 May 2012

(C) The Author(s) 2012. This article is published with open access at Springerlink.com

\begin{abstract}
Toxicokinetic-toxicodynamic (TKTD) models quantify the time-course of internal concentration, which is defined by uptake, elimination and biotransformation (TK), and the processes which lead to the toxic effects (TD). TKTD models show potential in predicting pesticide effects in fluctuating concentrations, but the data requirements and validity of underlying model assumptions are not known. We calibrated TKTD models to predict survival of Gammarus pulex in propiconazole exposure and investigated the data requirements. In order to assess the need of TK in survival models, we included or excluded simulated internal concentrations based on pre-calibrated TK. Adding TK did not improve goodness of fits. Moreover, different types of calibration data could be used to model survival, which might affect model parameterization. We used two types of data for calibration: acute toxicity (standard LC50, $4 \mathrm{~d}$ ) or pulsed toxicity data (total length $10 \mathrm{~d}$ ). The calibration data set influenced how well the survival in the other exposure scenario was predicted (acute to pulsed scenario or vice versa). We also tested two contrasting
\end{abstract}

Electronic supplementary material The online version of this article (doi:10.1007/s10646-012-0917-0) contains supplementary material, which is available to authorized users.

A.-M. Nyman $(\bowtie) \cdot$ K. Schirmer · R. Ashauer Department of Environmental Toxicology, Eawag,

Swiss Federal Institute of Aquatic Science and Technology,

Überlandstrasse 133, 8600 Dübendorf, Switzerland

e-mail: anna-maija.nyman@eawag.ch

A.-M. Nyman · K. Schirmer

Department of Environmental Systems Science, ETH Zürich, 8092 Zürich, Switzerland

K. Schirmer

EPF Lausanne, School of Architecture, Civil and Environmental

Engineering, 1015 Lausanne, Switzerland assumptions in ecotoxicology: stochastic death and individual tolerance distribution. Neither assumption fitted to data better than the other. We observed in 10-d toxicity experiments that pulsed treatments killed more organisms than treatments with constant concentration. All treatments received the same dose, i.e. the time-weighted average concentration was equal. We studied mode of toxic action of propiconazole and it likely acts as a baseline toxicant in G. pulex during 10-days of exposure for the endpoint survival.

Keywords Organism recovery - Delayed toxicity · Dose response model - Pesticide risk assessment Bioaccumulation

\section{Introduction}

Toxicokinetic-toxicodynamic (TKTD) models allow predicting pesticide effects on organisms in many exposure scenarios including fluctuating or pulsed concentrations (Ashauer et al. 2006; Ashauer et al. 2007a; Ashauer and Escher 2010; Brock et al. 2010; Jager et al. 2011; Mancini 1983; Péry et al. 2001). Toxicokinetics (TK) describe processes such as uptake, distribution, biotransformation and elimination while toxicodynamics (TD) describe the processes which lead to the effects after a compound reaches the sites of toxic action (McCarty and Mackay 1993). One of the advantages of TKTD models is the ability to predict survival of organisms upon pulsed exposure, as in pesticide applications. Aquatic environments are exposed to fluctuating pesticide concentrations, not only because pesticides are applied to fields repeatedly, but also due to natural processes which are influenced by weather (e.g. frequency and intensity of rainfall events), 
physico-chemical properties of the compound (e.g. hydrophobicity, stability), spills and non-agricultural point sources (Kreuger 1998; Wittmer et al. 2010).

The risks posed by a contaminant on aquatic organisms are estimated by relating the predicted environmental concentrations to effect levels (Brock et al. 2010; Traas and van Leeuwen 2007). The environmental concentrations are currently predicted using fate models (FOCUS 2001) but when comparing the exposure with the effects, different parts of the fate model output can be used, e.g. timeweighted average concentration (TWA) or initial peak concentration. However, using TWA concentrations might not always be protective for the effects of pulsed exposure. Several studies have indicated that toxic effects can be more severe in exposures to short pulses than in long constant exposure with the same TWA concentration (McCahon and Pascoe 1991; Parsons and Surgeoner 1991; Schulz and Liess 2000). The predicted environmental concentrations are compared with the effect level values such as predicted no effect concentration (PNEC) or effective concentration for $50 \%$ of individuals in a test group (EC50) (Brock et al. 2010; Traas and van Leeuwen 2007). These methods of estimating the effects are limited because the PNEC and EC50 values become meaningless in time-varying exposure patterns (Jager 2011). For example, the time which an organism needs to eliminate the substance and recover from the damage between contaminant pulses is ignored (Ashauer and Escher 2010; Jager 2011). TKTD models can simulate survival under fluctuating exposure and they take into account internal concentrations, organism damage and recovery. Therefore, they can overcome the problems related to predicted environmental concentrations, using the fate model output as it is, and predict effect levels by simulating the effects in the corresponding exposure pattern.

Still, uncertainties related to explaining and predicting the effects of pulsed exposure remain. Two assumptions, stochastic death (SD) and individual tolerance distribution (IT), have been proposed and there are no indications that only one of them would suit all combinations of chemicals and species (Ashauer and Brown 2008; Newman and McCloskey 2000; Zhao and Newman 2007). The stochastic death hypothesis assumes death to be a random process and all individuals have equal probability to die (Bedaux and Kooijman 1994; Jager et al. 2011; Newman and McCloskey 2000; Zhao and Newman 2007). The individual tolerance hypothesis, which has been dominating the ecotoxicological theory of survival, assumes that organisms have individual effect doses (Bliss 1935; Dauterman 1994; Newman and McCloskey 2000). The two hypotheses lead to different predictions of survival in subsequent pulses of equal concentrations-the SD assumption predicts equal mortality as during the previous pulse while the
IT assumption predicts no mortality during the second pulse because the individuals having low thresholds for effects were eliminated during the previous pulse. The recently developed TKTD model GUTS integrates SD and IT within one model (Jager et al. 2011). This model, including the damage, damage recovery and effect threshold, was used as a basis in the current study.

Aside from the uncertainty of the assumptions underlying SD or IT when applying TKTD models in risk assessment, there is also a lack of knowledge about the data requirements for model calibration. We conducted TK experiments, a standard 4 day LC50 test (lethal concentration for $50 \%$ of test animals) and a 10 day pulsed toxicity experiment on Gammarus pulex exposed to the fungicide propiconazole. The data were used to calibrate a set of TKTD models (Jager et al. 2011). The following model assumptions and options for calibration data were investigated: (a) how does the type of calibration data influence the parameter estimation and predictive power of the survival model, (b) how well does the survival model fit to data when the TK sub-model, simulating internal concentration, is included or excluded from the survival models, and (c) does the model assuming SD or IT better describe the data?

\section{Materials and methods}

\section{Gammarus pulex and propiconazole}

Gammarus pulex (Crustacea, Amphipoda, Gammaridae) is a key species in aquatic environments. It feeds on leaf and other organic material and therefore plays an important role in nutrient cycling (Anderson 1979). Many fish and other aquatic species feed on G. pulex and therefore G. pulex is an important part of food webs in European streams (MacNeil et al. 1997).

Propiconazole (CAS \#: 60207-90-1, $\log \mathrm{K}_{\mathrm{ow}}$ : 3.72) is an azole fungicide which inhibits the enzyme sterol $14 \alpha$ demethylase (Zarn et al. 2002). In fungi, the enzyme inhibition interferes with biosynthesis of ergosterol, which is an essential sterol component in fungal cell membranes. In animals, the enzyme is a part of the pathway leading to biosynthesis of cholesterol, which is a component of many other sterols (Zarn et al. 2002). In arthropods, one important group of cholesterol based hormones are ecdysteroids which are involved in molting: these hormones promote the replacement of the cutile (Lafont and Mathieu 2007). Therefore, even though propiconazole is a fungicide, it might act specifically in G. pulex. We studied the mode of toxic action of propiconazole by comparing internal concentrations of propiconazole in G. pulex with internal lethal concentrations $\left(\mathrm{ILC}_{50}\right)$ of known baseline toxicants in 
Daphnia magna (Maeder et al. 2004). If the internal concentrations in G. pulex fall into the range of ILC $_{50}$ values of baseline toxicants in $D$. magna, propiconazole is likely to act as a baseline toxicant (i.e. acts via narcosis) in G. pulex under chosen exposure conditions.

\section{Chemicals}

A mixture of ${ }^{14} \mathrm{C}$-labelled and unlabelled propiconazole was used. The unlabelled compound (chemical purity $98.4 \%)$ was purchased from Sigma-Aldrich and the labeled material (chemical purity $98.8 \%$, radiochemical purity $99.7 \%$ ) from the Institute of Isotopes Co., Ltd. Budapest, Hungary. The dosing mixture was dissolved in acetone.

\section{Handling of Gammarus pulex and exposure}

The test organisms were collected from a small headwater stream in the Itziker Ried, Switzerland (E 702150, $\mathrm{N}$ 2360850). To acclimatize them to laboratory conditions and equalize their nutritional status, G. pulex were maintained for 5-7 days prior to the experiments in a large aquarium in a temperature controlled room $\left(13{ }^{\circ} \mathrm{C}, 12: 12\right.$ light:dark photoperiod) and fed with horse chest-nut (Aesculus hippocastanum) leaves which were inoculated with the fungi Cladosporium herbarum (Naylor et al. 1989). The water in the aquarium was pre-aerated artificial pond water (APW, Table SI-1 in the Supporting Information) (Naylor et al. 1989).

In all experiments, ten test organisms were placed 1 day prior to the start of experiments in $600 \mathrm{~mL}$ beakers filled with $500 \mathrm{~mL}$ of APW. The beakers were covered with parafilm and kept in a climate chamber $\left(13{ }^{\circ} \mathrm{C}, 12: 12\right.$ light:dark photoperiod). The experiments started with dosing and subsequently, the water was stirred gently with a glass rod to distribute the chemical in experimental water (carrier acetone $<0.2 \%$ ). Propiconazole concentration in water was measured in every beaker directly after dosing (see below). Natural mortality and mortality caused by handling of the animals were measured using non-solvent (i.e. APW only) and solvent control beakers in addition to treated beakers. Inoculated horse-chestnut leaves were provided as leaf discs with a diameter of $20 \mathrm{~mm}$ and five leaf discs were given to organisms in each of the beakers. Eaten leaf discs were replaced with uncontaminated discs during the experiment. The organisms were transferred to beakers containing fresh uncontaminated APW and leaf discs, either to end an exposure period or to provide fresh APW at least once in 5 days. Water $\mathrm{pH}$, conductivity and oxygen concentration were measured regularly during experiments (Tables SI-2 to SI-5 in Supporting Information).
Design of TK experiments

The design of the TK experiments was based on Nuutinen et al. (2003) and Ashauer et al. (2010). Two TK experiments were conducted (TK1, TK2). Both included a 1-d exposure to propiconazole concentration of 7.8-9.5 nmol/mL which was below acute toxicity levels. After 1 day the animals were transferred to uncontaminated APW for 5-d (TK1) or 1-d (TK2). Eight replicate beakers were used, each containing ten G. pulex initially. Concentrations of propiconazole in medium were measured in every beaker (8) at different time points (TK1: 0, 5, 10, 24, 29, 34, 48, 72, 96 and $144 \mathrm{~h}$ and TK2: 0, 24, 29, 34 and $48 \mathrm{~h}$ ). The average concentrations of all eight beakers per sampling time were used for modelling (Tables SI-7 and SI-9 in Supporting Information).

To determine internal concentrations of propiconazole, G. pulex samples were taken at the same time points as the water samples; except no G. pulex samples were taken at time $0 \mathrm{~h}$. One $G$. pulex per beaker was taken each time, blotted dry with tissue paper and placed in a pre-weighed glass tube. Four G. pulex from different beakers were pooled into one sample, two pooled samples per sampling time were obtained. Pooled samples were weighed in preweighed glass tubes and frozen until analysis. The mean weight $( \pm$ SD) of pooled samples was $84.4 \pm 19.0 \mathrm{mg}$ ( $n=96$ G. pulex, 24 pooled samples) in experiment TK1 and $108.0 \pm 23.4 \mathrm{mg}$ ( $n=56$ G. pulex, 14 pooled samples) in experiment TK2. The weight of one individual was calculated by dividing the mass of one pooled sample, containing four individuals, by four (TK $1: 21.1 \pm 4.7 \mathrm{mg}$, TK 2: $27.0 \pm 5.9 \mathrm{mg}$ ).

\section{Design of TD experiments}

Acute toxicity of propiconazole in G. pulex was measured using a standard LC50 test design. The experiment consisted of seven pesticide concentrations between 8.2 and $37.4 \mathrm{nmol} / \mathrm{mL}$ (see Table SI-14 in Supporting Information) with two replicate beakers each, each beaker containing ten $G$. pulex initially. Propiconazole concentrations in water were measured and the survival of G. pulex was analysed by prodding and visual observation of movements daily for 4 days.

The pulsed toxicity test lasted 10 days and consisted of three treatments. Each of the treatments had seven replicate beakers, one non-solvent and one solvent control beaker. All beakers contained ten G. pulex initially. In two of the treatments (A, B), the organisms were exposed to two 1-d pulses (concentration around LC30, $28 \mathrm{nmol} / \mathrm{mL}$ ). Between pulses, the organisms had a 2-d (A) or 6-d (B) period to recover in uncontaminated APW. In the third treatment $(\mathrm{C})$, the animals were exposed constantly to the same time-weighted average (TWA) concentration as in the pulsed treatments $(4.6 \mathrm{nmol} / \mathrm{mL})$. Treatment $\mathrm{C}$ was 
conducted not only to compare the toxic effects of pulsed exposure with the corresponding constant TWA concentration but also to maximize information content for calibration of the TKTD models (Albert et al. 2012). The propicanozole concentration in water was measured and the survival was observed on a daily basis.

\section{Determination of aqueous chemical concentrations}

In all experiments, aqueous concentrations were measured daily or more often (see sections above). A volume of $1 \mathrm{~mL}$ was sampled from experimental waters, $10 \mathrm{~mL}$ of Ecoscint A scintillation cocktail (Chemie Brunschwig, Switzerland) was added, samples were shaken and measured using a liquid scintillation counter (LSC, Tri-Carb 2200CA, Packard, USA). The counts were corrected for background activity by subtracting the activities in $10 \mathrm{~mL}$ Ecoscint A combined with $1 \mathrm{~mL}$ of uncontaminated experimental water (control beakers).

\section{Determination of internal concentrations}

For analysis, the frozen animals were homogenized in test tubes using a glass rod. Methanol was added twice during homogenization ( 1 and $2.5 \mathrm{~mL}$ ). Then, the tubes were placed into an ultrasonic bath for $5 \mathrm{~min}$ and the homogenate was filtered through a $0.2 \mu \mathrm{m}$ syringe filter (regenerated cellulose). The homogenate, syringe and filter were washed two times with methanol by vortexing. The filtrate was concentrated to a suitable volume $(90 \mu \mathrm{L})$ using GeneVac (EZ-2 PLUS, Genevac, UK) with a method of low boiling point, $60{ }^{\circ} \mathrm{C}$, for $50 \mathrm{~min}$, and under nitrogen flow. Nanopure water was added to obtain a total volume of $300 \mu \mathrm{L}$ to establish the appropriate methanol-water ratio for high-performance liquid chromatography (HPLC) analysis of propiconazole (see Table SI-6 in Supporting Information). The samples were split and in one aliquot the concentrations were measured using a HPLC (HP 1100, Agilent) with a radiodetector (500 TR, Packard) in order to detect both parent propiconazole and its metabolites. The other aliquot was analysed with the LSC to measure the recovery of the HPLC. The recovery was on average $93 \pm 18 \%$. In addition, the overall recovery of the sample preparation and quantification was obtained from samples of control G. pulex spiked with known amounts of ${ }^{14} \mathrm{C}$ labelled propiconazole. The overall recovery was $72-91 \%$.

Model design, formulation and description

\section{TK model}

Both the toxicokinetic (TK) and toxicodynamic (TD) models used here assume that the organisms do not change during the experiments. They are considered as one compartment, thus the chemical is assumed to be evenly distributed throughout the organism. A one compartment model (Eq. 1) was used to simulate TK. For this, uptake $\left(k_{\text {in }}\right)$ and elimination $\left(k_{\text {out }}\right)$ rate constants were estimated from TK data.

$\frac{\mathrm{d} C_{\text {int }}(t)}{\mathrm{d} t}=C_{\text {ext }}(t) \cdot k_{\text {in }}-C_{\text {int }}(t) \cdot k_{\text {out }}$

where $C_{\text {int }}(\mathrm{t})$ is the internal propiconazole concentration in organisms $[\mathrm{nmol} / \mathrm{g}], C_{\text {ext }}(\mathrm{t})$ is the concentration in water $[\mathrm{nmol} / \mathrm{mL}], \quad k_{\text {in }}$ is the uptake rate constant $\left[\mathrm{mL} \mathrm{g}^{-1} \mathrm{~d}^{-1}\right], k_{\text {out }}$ is the elimination rate constant $[1 / \mathrm{d}]$ and $t$ is time [d].

\section{Survival models}

Survival modelling was based on Jager et al. (2011). Two models assuming SD or IT were compared when pulse toxicity data, acute toxicity data or both were used to calibrate the models. SD models have one value for the threshold of survival and after exceeding it, an organism has an increased probability to die. In contrast, according to IT models the threshold is distributed within the population and death is instantaneous after exceeding the individual threshold. Both models were calibrated including and excluding the pre-calibrated TK model (full-SD, full-IT models and reduced-SD, reduced-IT models). An illustration of model types is given in Fig. 1.

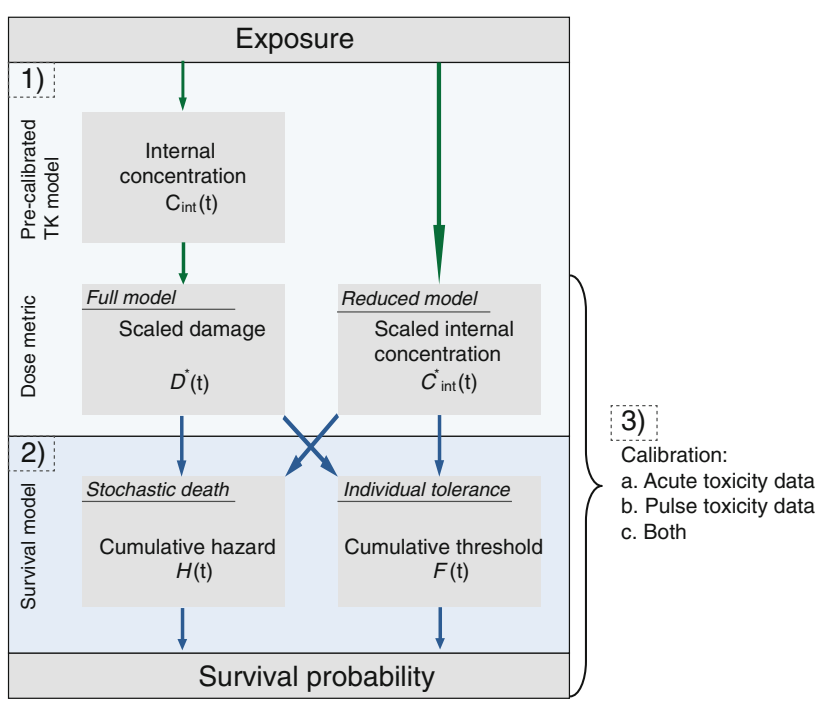

Fig. 1 Structure of TKTD models used in this study. We tested three variations of model assumptions and data needs (numbers 1-3). First, the need of a toxicokinetic (TK) submodel was tested (1). Second, the assumption of survival being stochastic or deterministic for an individual was studied (2). Third, the data needed for calibration of the survival model was investigated (3) 


\section{Stochastic death models}

Implementation of the stochastic death models is given in Eqs. 2-7. First (2) and (3) were used to calculate the cumulative hazard at time $t(H(t))$ when TK parameters $k_{\text {in }}$ and $k_{\text {out }}$ were included (full-SD). Equation 1 was used to estimate uptake and elimination rate constants prior to survival modelling by fitting Eq. 1 to the TK data alone. Then, the pre-calibrated TK model was used to simulate the internal concentrations $\left(C_{\text {int }}\right)$ in the survival model. Including TK in the survival model enabled the use of an explicitly modeled damage stage as dose metric for the survival probability. Thus, the TK and TD recovery processes could be distinguished, i.e. $k_{\mathrm{d}}$ in Eqn 2 describes solely the TD recovery, the elimination rate constant being estimated in Eq. 1.

$\frac{\mathrm{d} D^{*}(t)}{\mathrm{d} t}=k_{\mathrm{d}} \cdot\left(C_{\mathrm{int}}(t)-D^{*}(t)\right)$

$\frac{\mathrm{d} H(t)}{\mathrm{d} t}=k_{\mathrm{k}} \cdot \max \left(D^{*}(t)-z, 0\right)+h_{\mathrm{b}}(t)$

where $D^{*}(\mathrm{t})$ is the scaled damage $[\mathrm{nmol} / \mathrm{g}], k_{\mathrm{d}}$ is the damage recovery $[1 / \mathrm{d}], \quad k_{\mathrm{k}}$ is the killing rate $\left[\mathrm{g} \mathrm{nmol}^{-1} \mathrm{~d}^{-1}\right], H(\mathrm{t})$ is the cumulative hazard of an individual $[-], z$ is the threshold for effects $[\mathrm{nmol} / \mathrm{g}], h_{\mathrm{b}}$ is the background hazard rate [1/d] (Eq. 6) and the 'max' function selects the maximum of either 0 or $\left(D^{*}(\mathrm{t})-z\right)$.

Toxicokinetics can be excluded in survival models (reduced-SD, Eqs. 4-5), but a slightly different formulation of the TD concept is needed when compared to the full-SD model (Eqs. 2-3). As the actual internal concentrations are unknown, the scaled internal concentration is used as the dose metric for the survival model (see Jager et al. 2011 for detailed explanations) and therefore the model does not include the damage stage explicitly. Instead, the dominant rate constant $k_{\mathrm{d}}$ describes both compensating processes, TK elimination and TD damage recovery. The slowest of these processes will dominate the value of $k_{\mathrm{d}}$.

$\frac{\mathrm{d} C_{\mathrm{int}}^{*}(t)}{\mathrm{d} t}=k_{\mathrm{d}} \cdot\left(C_{\mathrm{ext}}(t)-C_{\mathrm{int}}^{*}(t)\right)$

$\frac{\mathrm{d} H(t)}{\mathrm{d} t}=k_{\mathrm{k}} \cdot \max \left(C_{\mathrm{int}}^{*}(t)-z, 0\right)+h_{\mathrm{b}}(t)$

where $\left.C_{\mathrm{int}}^{*} \mathrm{t}\right)$ is the scaled internal concentration $[\mathrm{nmol} / \mathrm{mL}]$, $k_{\mathrm{d}}$ is the dominant rate constant $[1 / \mathrm{d}], k_{\mathrm{k}}$ is the killing rate $\left[\mathrm{mL} \mathrm{nmol}{ }^{-1} \mathrm{~d}^{-1}\right.$ ] and $z$ is the threshold for effects [nmol/ $\mathrm{mL}]$.

The background hazard rate $h_{\mathrm{b}}$ was obtained by fitting Eq. 6 to survival data of non-solvent and solvent controls combined.

$S_{\mathrm{b}}=e^{-h_{\mathrm{b}} t}$ where $S_{\mathrm{b}}$ is the background survival probability [-] describing survival in unexposed conditions.

Once the cumulative hazard $H(t)$ is obtained either in the reduced or full-SD model, the survival probability, $S(\mathrm{t})$ [-], was calculated using Eq. 7.

$S(t)=e^{-H(t)}$

\section{Individual tolerance models}

The model that assumes the threshold for death to be drawn from an individual tolerance distribution is presented in Eqs. 8-10. Reduced- and full-IT models use the same dose metrics as in SD models, scaled internal concentration $C_{\text {int }}^{*}$ (reduced model, Eq. 4) and scaled damage $D^{*}$ (full model, Eq. 2). Cumulative threshold distributions are based on a log-logistic cumulative distribution function (Eq. 8 for full model and Eq. 9 for reduced model). The resulting survival probability is given by Eq. 10 .

$F(t)=\frac{1}{1+\left(\max _{0<\tau<t} C_{\mathrm{int}}^{*}(\tau) / \alpha\right)^{-\beta}}$

$F(t)=\frac{1}{1+\left(\max _{0<\tau<t} D^{*}(\tau) / \alpha\right)^{-\beta}}$

$S(t)=(1-F(t)) \cdot e^{-h_{\mathrm{b}} t}$

where $F(\mathrm{t})$ is the log-logistic cumulative distribution function for the threshold $[-], \alpha$ is the median of the distribution [units of dose metric, either $\mathrm{nmol} / \mathrm{mL}$ for Eq. 8 or $\mathrm{nmol} / \mathrm{g}$ for Eq. 9], $\beta$ determines the width of the distribution $[-]$ and the 'max' function selects the largest value of the dose metric $C^{*}$ or $D^{*}$ that occurred until time $t$.

\section{Model calibration}

Both, the models for SD and IT were calibrated using pulse toxicity data alone, acute toxicity data alone or both data sets together. A two-step calibration was carried out. First a least squares fit using the Marquardt algorithm yielded parameter estimates. These served as initial values in the second step where the log-likelihood function (Eq. 11) (Jager et al. 2011) was maximized to find the final best fit values.

$\ln l(\theta \mid y)=\sum_{i=1}^{n+1}\left(y_{i-1}-y_{i}\right) \ln \left(S_{i-1}(\theta)-S_{i}(\theta)\right)$

where, $l$ is the likelihood for the vector of parameters $\theta$ given the observations $y$ and $y$ is the time series of the number of survivors $\left(y_{0} \ldots y_{\mathrm{n}}\right)$.

The likelihood function compares the observed number of death events in an observation interval with the death 
events predicted by the model (Jager et al. 2011). Therefore, maximising the likelihood function yields the parameter set that best describes the death events over time assuming independent death events. The log-likelihoods of the treatments were added to obtain the total likelihood. The profile of log-likelihoods was used to obtain the confidence intervals $(95 \%)$ for each of the parameters (Kooijman and Bedaux 1996). Modelling procedures, including run settings and initial values, are described in more detail in the SI.

In order to estimate the relative goodness of fit amongst the models and calibration data, the log-likelihood values were compared. We use the term 'goodness of fit' not only when the model was fitted to data by adjusting parameter values, but also when a combination of model and parameter set was used to simulate survival in another exposure scenario. Then the likelihood value was obtained by comparing the prediction with independent observations. To compare the simulation performance of each model easily between data sets and model types, we added the likelihood value of the simulation to that of the fit (shown in Fig. 4 as combined likelihood value above each model type). This is called total likelihood in the following text. In addition, the mean percentage error (MPE) was calculated (Eq. 12), because that corresponds to a practitioners view on model performance.

MPE $=\frac{1}{n} \sum \frac{\left|S_{\text {obs }}-S_{\text {model }}\right|}{S_{\text {model }}} \cdot 100$

where MPE is the mean percentage error of the fraction of survivors [\%], $\mathrm{S}_{\mathrm{obs}}$ is the observed fraction of survivors, $\mathrm{S}_{\text {model }}$ is the model prediction of the fraction of survivors and $\mathrm{n}$ is the number of data points used in the calculation.

Survival curves in the pulsed exposure experiment were compared using the Kaplan-Meier log-rank test. The method uses the survival curves over time and compares them pairwise (e.g. Control vs Treatment $\mathrm{C}$ ). It generates a $p$ value testing the hypothesis that the survival curves are identical in the overall population.

\section{Model implementation}

The software GraphPad Prism 4.03 (GraphPad Software Inc., San Diego, USA) was used for determination of 1-d, 2-d, 3-d and 4-d LC50 values from acute toxicity data (Least squares optimization to sigmoidal dose-response model, top fixed at $100 \%$ and bottom fixed at $0 \%$ ) and for comparison of the survival curves in the pulsed exposure experiment (Kaplan-Meier log-rank test). For TK and TD modelling the software ModelMaker 4 (Cherwell Scientific Ltd., Oxford, UK) was used. The maximum likelihood search was implemented by minimizing-(sum of loglikelihoods, Eq. 11). The TRACE (transparent and comprehensive ecological modelling) documentation (Schmolke et al. 2010) was followed in the modelling work and is provided in the supporting information (Box SI-1).

\section{Results}

\section{Toxicokinetics}

Two possible propiconazole metabolites were observed (see Figs SI-1 and SI-2 in supporting information). Metabolite 1 appeared in 14 samples out of total 30 samples, but only in three samples was the concentration above the minimal detectable amount (MDA). The metabolite two appeared in eight samples and in none of them was the concentration above the MDA. The MDA for 1 min peaks was $78.8 \mathrm{dpm}$. As the metabolites remained mostly below levels of quantification, they were not identified. We used only the peaks of the parent compound as input for the TK model because we cannot model metabolite kinetics using only three samples. Thus $k_{\text {out }}$ denotes the loss of parent propiconazole, which can occur not only via excretion or diffusion (i.e. elimination) but also via biotransformation into metabolites. Based on the TK modelling, the uptake rate constant $k_{\text {in }}$ was $130.9 \pm 21.9 \mathrm{~L} /(\mathrm{kg} / \mathrm{d})$ and the elimination rate constant $k_{\text {out }}$ was $6.9 \pm 1.2[1 / \mathrm{d}]$. The time when $95 \%$ of propiconazole is eliminated was calculated as 0.43 days (around $10 \mathrm{~h}$ ). By dividing $k_{\text {in }}$ by $k_{\text {out }}$, the bioaccumulation factor (BAF) can be calculated, even without reaching steady state in the experiment. Based on this, propiconazole has a BAF of $19 \mathrm{~L} / \mathrm{kg}$. Dividing the internal concentration of the parent compound $[\mathrm{nmol} / \mathrm{g}]$ by its external concentration $[\mathrm{nmol} / \mathrm{mL}]$ after 1 -d exposure yields a BAF of $22 \mathrm{~L} / \mathrm{kg}$. The time courses of external and internal concentrations are illustrated in Fig. 2 and the raw data are provided in the SI. The mortality during the TK tests was low, around $2 \%$ in exposed and control beakers.

\section{Toxicodynamics}

Based on the acute toxicity test, the LC50 values (lowerupper $95 \%$ confidence limit) were estimated as follows: 34.5 (26.5-45.0) nmol/mL after 1-d exposure, 22.5 (20.9-24.3) $\mathrm{nmol} / \mathrm{mL}$ after 2 -d exposure, 19.6 (18.3 to 21.0$) \mathrm{nmol} / \mathrm{mL}$ after 3-d exposure and $19.2(17.6-20.9) \mathrm{nmol} / \mathrm{mL}$ after $4-\mathrm{d}$ exposure. Dose-response curves are provided in Fig SI-3 (in supporting information). In the pulse toxicity experiment, the mortality directly after the first pulse was around $20(\mathrm{Tr}$. B) to 30 (Tr. A) \%, while after the second pulse, it was only 8 ( $\operatorname{Tr}$ A) to 9 (Tr B) \% (Fig. 3). Altogether, the time-weighted average concentration did not kill as many individuals as the pulse treatments. Survival at the end of treatment A was $51 \%$, treatment B $53 \%$ and in treatment C (TWA 

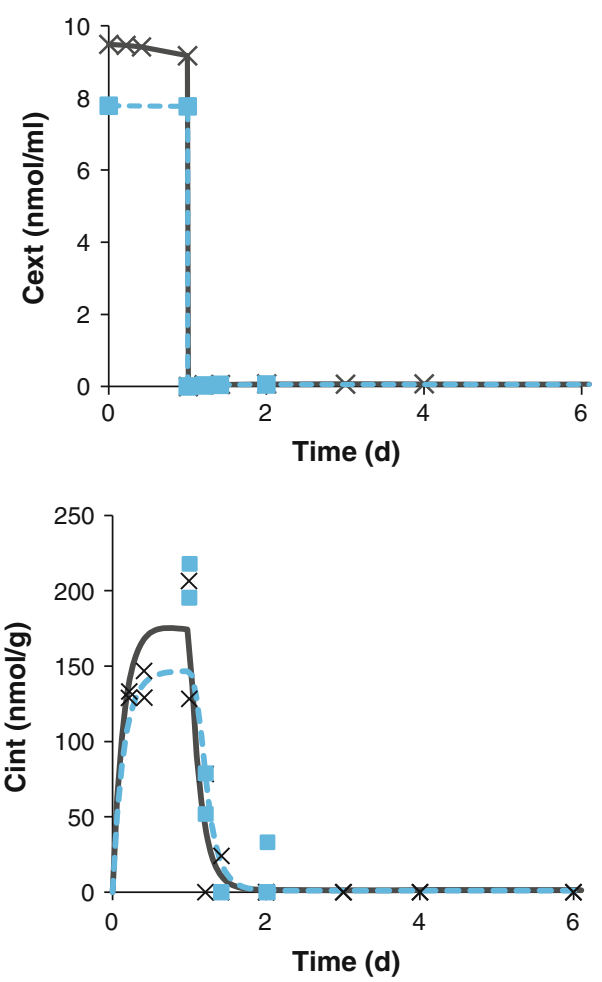

Fig. 2 External concentration $\left(C_{\text {ext }}\right)$ and internal $\left(C_{\text {int }}\right)$ concentration of propiconazole in Gammarus pulex; measured in two separate toxicokinetic experiments (TK 1: crosses, TK 2: squares). The toxicokinetic model was calibrated using both data sets simultaneously (TK 1: solid line, TK 2: dashed line). Only the concentration of parent compound was used

concentration) $77 \%$ of the animals survived. Based on a survival curve analysis (Kaplan-Meier log-rank test), the differences between the treatment $\mathrm{C}$ and treatments $\mathrm{A}$ and $\mathrm{B}$ were significant $(p<0.01)$. In fact, the survival in treatment $C$ did not differ significantly from that of the controls $(p=0.06)$. The survival of the controls was $90 \%$ in the 10-d pulse toxicity experiment and $95 \%$ in the 4-d acute toxicity test. The raw data of the pulse toxicity experiment and acute toxicity experiment, including measured exposure concentrations and number of alive organisms during the time course of the experiments, are provided in Tables SI-11 to SI-14.

Estimates of the parameters $k_{d}, k_{\mathrm{k}}, z, \alpha$ and $\beta$ are provided in Table 1. All models (reduced-SD, full-SD, reduced-IT, full-IT) with their intermediate steps are illustrated in Fig. 3. Altogether, the SD models were difficult to calibrate using pulse toxicity data alone and therefore a modified initial parameter set and run settings were used (see Table SI-15). In addition, this combination of model and calibration data resulted in very different parameter estimates when compared with other models (Table 1). However, it cannot be concluded that the IT models better fit the data (Figs. 3, 4). A comparison of goodness of fits of the models and calibration data sets is provided in Fig. 4.

\section{Discussion}

Mode of action of propiconazole in G. pulex

Propiconazole inhibits the enzyme sterol $14 \alpha$-demethylase which is acting in the pathway that leads to biosynthesis of cholesterol in animals and might thus interfere with molting of invertebrates (Lafont and Mathieu 2007; Zarn et al. 2002). Here, the mode of toxic action of propiconazole was studied by comparing simulated internal concentrations of propiconazole in G. pulex in our toxicity tests with $\mathrm{ILC}_{50}$ of known baseline toxicants in Daphnia magna (Maeder et al. 2004). The TK model was used to simulate the internal concentrations in toxicity experiments. The concentrations were corrected by lipid content, i.e. by dividing the total concentration by the amount of lipids (G. pulex: lipid content of $1.5 \%$, which was measured by a gravimetric method, unpublished data; D. magna: lipid content of $1.7 \%$ (Kretschmann et al. 2011)). The simulated internal concentrations in G. pulex reached the lower range of the baseline toxicant ILC $_{50}$ of D. magna both, in the pulsed toxicity treatments, and in the two highest concentrations of the acute toxicity test (treatments A and B) (Figs. SI-5 and SI-6 in supporting information). When the lower range of baseline $\mathrm{ILC}_{50}$ was reached, mortality of $20 \%$ (pulsed toxicity test, treatment $\mathrm{B}$, concentration around LC30) to $50 \%$ (acute toxicity test, treatments A and B) was observed in our experiments. In the TWA concentration of the pulsed toxicity experiment, the internal concentrations remained far below the $\mathrm{ILC}_{50}$ range and accordingly, the survival curve of the treatment did not differ from that of the control. Only the second pulse in both pulsed treatments caused lower mortality than $20 \%$, even though simulated internal concentration reached the $\mathrm{ILC}_{50}$ range of the baseline toxicants. Internal lethal concentration can be calculated as BCF $\times$ LC50 (Maeder et al. 2004; McCarty and Mackay 1993), which was $43.7 \mu \mathrm{mol} / \mathrm{g}$ lipid for propiconazole in G. pulex. This falls into the D. magna ILC range of baseline toxicants (Maeder et al. 2004) corrected by lipid content of $1.7 \%$ (Kretschmann et al. 2011), 35-312 $\mu \mathrm{mol} / \mathrm{g}$ lipid. Altogether, propiconazole seems to act as a baseline toxicant in G. pulex in $10 \mathrm{~d}$ exposure for the endpoint survival. However, uncertainty remains because the baseline toxicant values that we compare with are for D. magna and the internal propiconazole concentrations reached only the lower $\mathrm{ILC}_{50}$ ranges. 


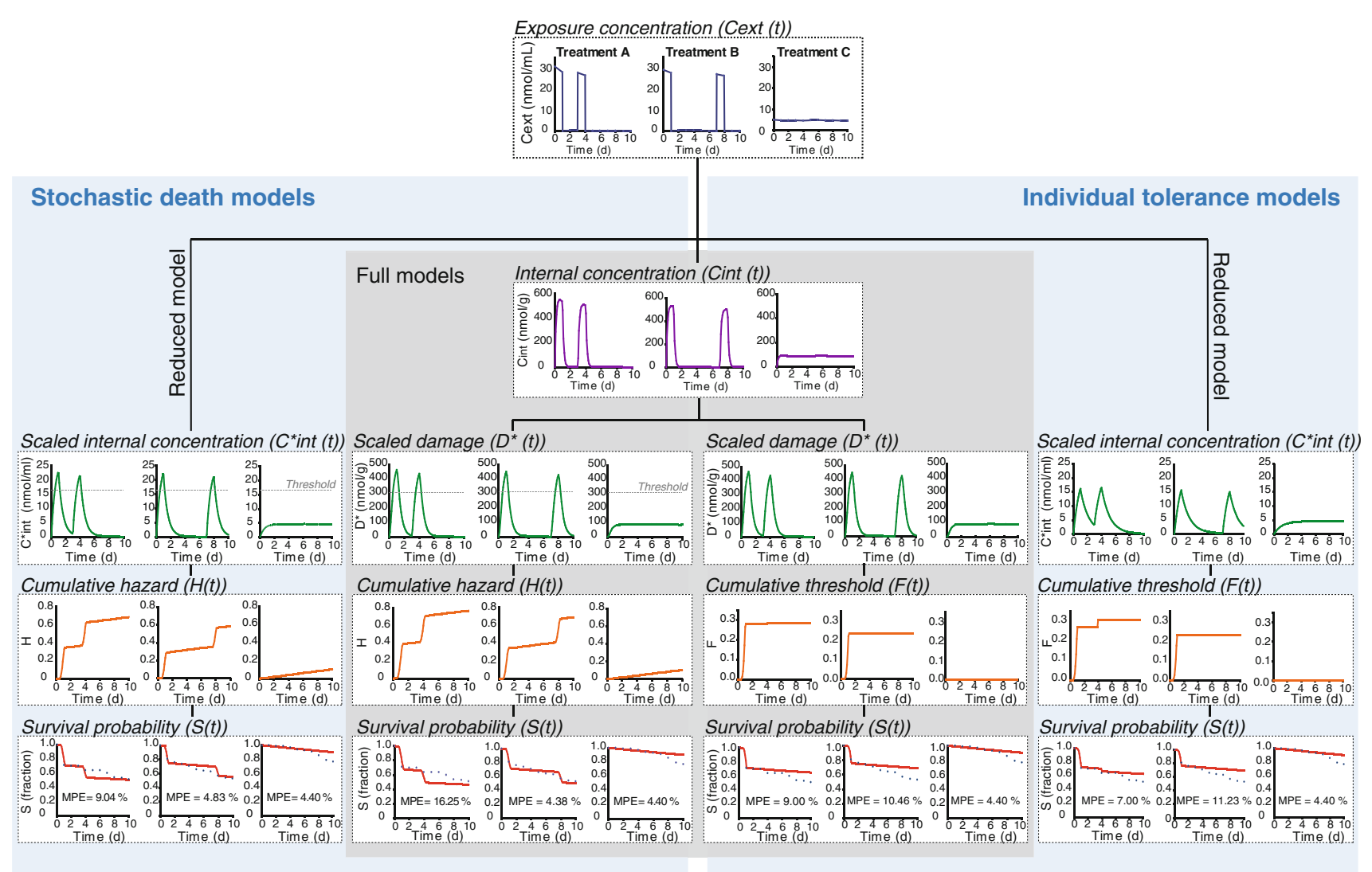

Fig. 3 The stochastic death and individual tolerance models describing survival of Gammarus pulex in response to propiconazole exposure. Models are described step-by-step, starting from exposure concentrations, followed by internal concentration (full models) and dose metric illustration (scaled internal concentration/scaled damage) and survival model (cumulative hazard $\mathrm{H} /$ cumulative threshold $\mathrm{F}$ and

\section{Effects of pulsed exposure}

In our pulsed toxicity experiment, the treatments with two propiconazole pulses killed more individuals than the constant treatment with the corresponding TWA concentration. It has been hypothesised that the high exposure peaks cause higher bioaccumulation and thus more severe toxic effects than lower constant exposure with equivalent daily mean concentrations (Curtis et al. 1985; Parsons and Surgeoner 1991). This has been observed by Curtis and co-authors (1985) when they exposed early life-stage steelhead trout to fenvalerate (Curtis et al. 1985). Concentration dependent bioaccumulation (Liu et al. 2011) might also explain the results of this study, although we do not have direct evidence to support that hypothesis. However, permethrin caused more mortality to A. aegypti when the exposure was pulsed even though the exposure concentrations were equivalent. Thus, differing exposure concentrations could not explain the higher mortality by higher bioaccumulation in pulsed exposure (Parsons and Surgeoner 1991). Instead, organisms might have partially eliminated the compound survival fraction $\mathrm{S}$ ). The dots in survival probability figures represent measured data in the pulse toxicity experiment. MPE (\%) in survival graphs denotes mean percentage error. The models were calibrated using both acute and pulse toxicity data sets, however, only pulsed toxicity data is shown here (for a fit to acute toxicity data, see Fig. SI-4 in supporting information)

and recovered between the pulses which enabled them to eat and thus take up more permethrin during the following contaminant pulse, while immobility prevented the animals from eating in constant exposure (Parsons and Surgeoner 1991; Reinert et al. 2002).

Predicted patterns of survival in multiple pulse exposures are different when based on different hypotheses of survival, stochastic death and individual tolerance (Ashauer 2010; Jager et al. 2011; Newman and McCloskey 2000; Zhao and Newman 2007). TKTD models can be used to study whether pesticide induced mortality supports IT or SD. We observed in this study with propiconazole that there appeared no clear trend between goodness of fits of SD and IT models (Fig. 4). The IT models seemed to fit better to the treatment with short recovery time between the exposure pulses while SD models described the treatment with a longer recovery period better (Fig. 3). Therefore, the hypothesis of either individual tolerance distribution or stochastic death might not solely explain the toxicity in subsequent pulses as it has been observed also by other authors (Newman and McCloskey 2000). 
Table 1 Estimates of toxicodynamic parameters (lower-upper 95\% confidence limit) for Gammarus pulex and propiconazole according to different survival models

\begin{tabular}{|c|c|c|c|c|c|c|c|}
\hline \multicolumn{2}{|c|}{ Model } & \multirow{2}{*}{$\begin{array}{l}\text { Calibration data } \\
\text { Pulsed toxicity }\end{array}$} & \multirow{2}{*}{$\frac{k_{\mathrm{d}}^{\mathrm{a}}}{14.5(4.9-\text {-n.d. })}$} & \multirow{2}{*}{$\frac{k_{\mathrm{k}}^{\mathrm{b}}}{0.0005(0.0004-0.0007)}$} & \multirow{2}{*}{$\frac{z^{c}}{73.2(31.8-81.7)}$} & \multirow{2}{*}{$\frac{\alpha^{\mathrm{d}}}{-}$} & \multirow{2}{*}{$\frac{\beta^{\mathrm{e}}}{-}$} \\
\hline SD & Full & & & & & & \\
\hline & & Acute toxicity & $2.7(2.2-3.6)$ & $0.0073(0.0055-0.0094)$ & $316.1(302.2-325.2)$ & - & - \\
\hline & & Both & $2.3(2.1-2.7)$ & $0.0051(0.0042-0.0062)$ & $311.6(301.0-323.3)$ & - & - \\
\hline & Reduced & Pulsed toxicity & $5.1(2.6-12.3)$ & $0.0096(0.0070-0.0125)$ & $3.2(1.7-4.1)$ & - & - \\
\hline & & Acute toxicity & $2.1(1.7-2.6)$ & $0.1339(0.1012-0.1724)$ & $16.6(15.8-17.1)$ & - & - \\
\hline & & Both & $1.7(1.5-1.8)$ & $0.1260(0.1031-0.1534)$ & $16.4(15.8-16.9)$ & - & - \\
\hline \multirow[t]{6}{*}{ IT } & Full & Pulsed toxicity & $0.6(0.4-0.7)$ & - & - & $341.1(292.8-400.2)$ & $2.4(1.9-3.3)$ \\
\hline & & Acute toxicity & $1.0(0.8-1.1)$ & - & - & $343.8(331.5-386.2)$ & $6.4(5.3-9.3)$ \\
\hline & & Both & $1.0(0.9-1.1)$ & - & - & $364.4(350.4-378.4)$ & $7.6(6.1-9.3)$ \\
\hline & Reduced & Pulsed toxicity & $0.4(0.3-0.5)$ & - & - & $16.1(12.2-17.8)$ & $2.2(1.5-2.6)$ \\
\hline & & Acute toxicity & $0.9(0.6-1.0)$ & - & - & $18.2(17.7-21.0)$ & $6.5(5.4-9.3)$ \\
\hline & & Both & $0.8(0.8-0.9)$ & - & - & $18.7(18.0-19.4)$ & $7.4(6.1-9.2)$ \\
\hline
\end{tabular}

SD Stochastic death model, IT Individual tolerance model, Full = Model including toxicokinetics, Reduced = Model excluding toxicokinetics n.d. not determined (no upper limit found, must be $>40$ )

${ }^{a}$ Damage recovery [1/d] (full models) or the dominant rate constant [1/d], which describes both compensating processes, TK elimination and TD damage recovery, but the slowest process dominates the value (reduced models)

${ }^{\mathrm{b}}$ Killing rate $\left[\mathrm{mL} \mathrm{nmol}{ }^{-1} \mathrm{~d}^{-1}\right.$ or $\mathrm{g} \mathrm{nmol}{ }^{-1} \mathrm{~d}^{-1}$ depending on the dose metric]

c Threshold for effects [nmol/mL or nmol/g depending on the dose metric]

${ }^{\mathrm{d}}$ Median of threshold distribution [nmol/mL or $\mathrm{nmol} / \mathrm{g}$ depending on the dose metric]

e Width of the distribution [-]

There are other relevant processes which might affect survival (see below) and they might cause deviations from predictions provided by either of the hypothesis.

On one hand, the first pulse might weaken the surviving organisms and decrease their health, leading to increased mortality within the next pulse (Reinert et al. 2002). However, from our data we cannot infer whether the second pulse lead to "increased mortality", because we come to opposing conclusions depending on whether we assume IT or SD (yes for IT, no for SD). In addition, our TD models would accommodate such increasing damage level. On the other hand, a first pulse might decrease mortality in the next pulse by acclimatizing the organisms to the chemical stress. For example, the first pulse might induce biotransformation and detoxification enzymes, which help the organism to deal with the subsequent pulses, and the organism might undergo some changes which alter the chemical's target site between the pulses (Dauterman 1994; Reinert et al. 2002). Acclimatization might interfere with the two extreme hypotheses of survival and therefore neither the SD nor the IT model alone could explain the pesticide induced mortality in a pulsed exposure scenario. However, extreme cases of SD or IT could explain survival patterns in several studies. For example, a pesticide inhibiting acetylcholine esterase, diazinon, has shown clear stochastic death patterns in G. pulex (Ashauer et al. 2010). In addition, data of mosquitofish exposed to sodium chloride or pentachlorophenol pulses supported that the stochastic component determined fish survival (Newman and McCloskey 2000). On the other hand, the support for individual tolerance theory originates in observations that insects after few generations seemed to achieve resistance to herbicides and insecticides (Bliss 1935; Dauterman 1994). Mode of toxic action as well as species characteristics might also affect the applicability of the stochastic death or individual tolerance hypothesis.

The differences between SD and IT are reflected in organism recovery times, which have been shown to be important in determining the effects of pulsed exposure (Ashauer et al. 2010; Kallander et al. 1997). Here, we calculated organism recovery times, which are defined as the time when the damage level in the organism has dropped to $5 \%$ of the maximum after a defined pulse (Ashauer et al. 2007b; Ashauer et al. 2010). The recovery time was less than 3 days in all SD models but ranged from 4.2 to 8.5 days according to the IT models (Table 2). The differences between SD and IT are related to different model assumptions, i.e. according to IT models, organisms should not be recovered from the previous contaminant pulses in order to produce mortality during subsequent ones.

Organism recovery can be driven either by TK (i.e. elimination) or TD (i.e. damage recovery). Propiconazole was eliminated shortly after transfer to uncontaminated water $(95 \%$ elimination time $\approx 10 \mathrm{~h}$ ). When comparing 
Fig. 4 Goodness of fits among all model types and calibration data sets. 'Full model' denotes a model including toxicokinetics while 'reduced model' refers to a model excluding toxicokinetics. The models were calibrated with different data sets (acute or pulsed toxicity data or both). The observed fraction of survivors is plotted against the predicted survival. Mean predicted error (MPE, \%) and likelihood values are provided above each plot. The maximum log-likelihood was implemented by minimizing(sum of likelihoods) and therefore the smaller the value is the better is the fit

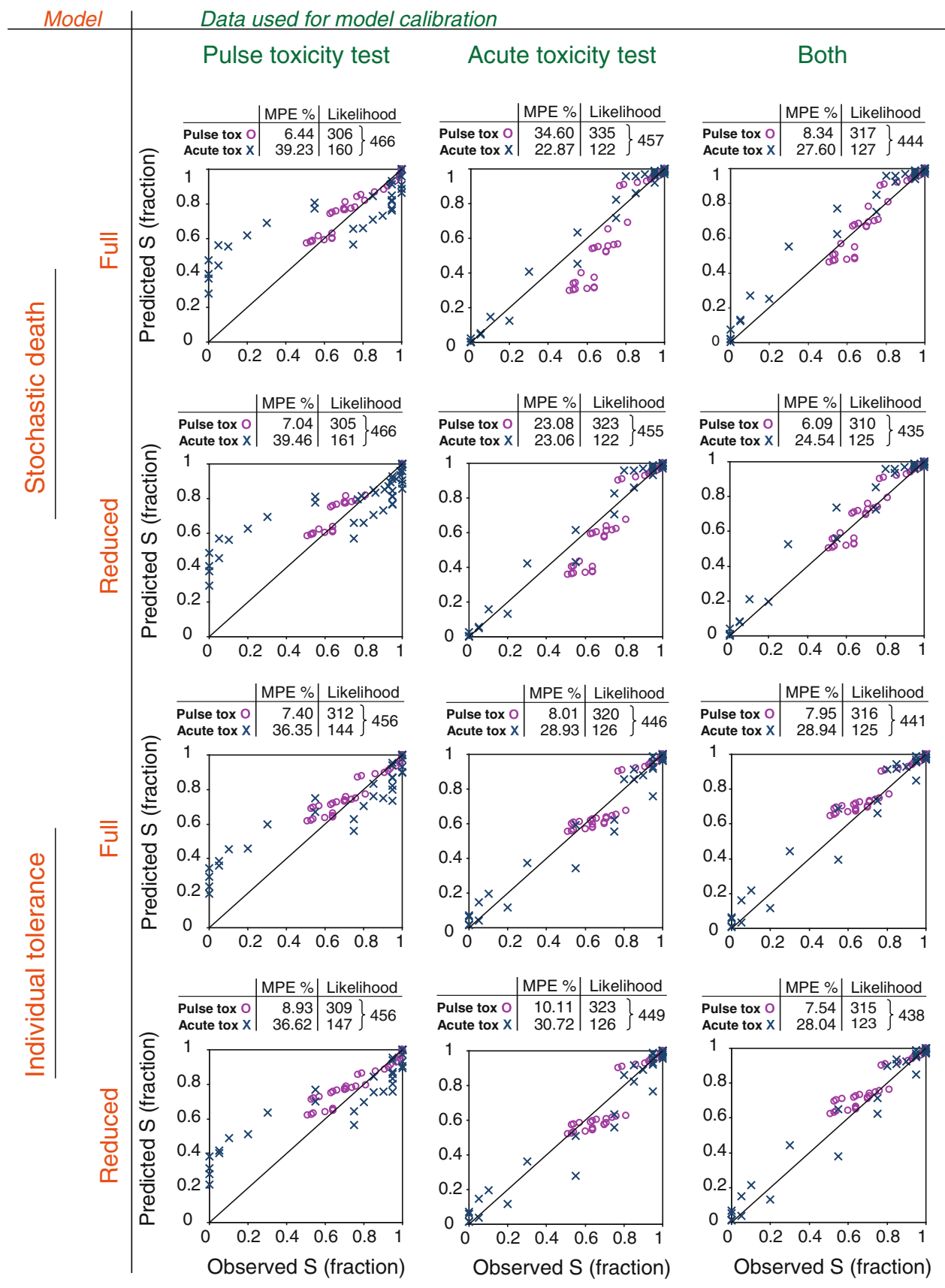

the TK and TD recovery parameters $k_{\text {out }}$ and $k_{\mathrm{d}}$, the TD recovery rate constant $k_{\mathrm{d}}$ was lower than the elimination rate constant $k_{\text {out }}$ according to almost all models (Table 1). Therefore, it can be concluded that TD recovery dominated overall organism recovery. Ashauer et al. (2010) came to the same conclusion when they exposed G. pulex to diazinon. The opposite has been observed by Ashauer et al. (2007b) when G. pulex were exposed to carbaryl: the elimination rate constant is $0.27(1 / \mathrm{d})$ while TD recovery rate constant is $0.97(1 / \mathrm{d})$ (Ashauer et al. 2007b), although this observation must be revised in light of new insights into biotransformation, also of carbaryl, in $G$. pulex
(Ashauer et al. 2012). Ashauer et al. (2007b) measured only total radioactivity, not biotransformation. When biotransformation to naphthol-sulphate is also considered, the elimination rate of carbaryl is 2.3 (1/day) and the total loss rate for carbaryl is $5.6(1 / \mathrm{d})$ (Ashauer et al. 2012). Thus, the organism recovery of $G$. pulex exposed to carbaryl is also dominated by toxicodynamics.

Data requirements

Previously it has been claimed that TK is an essential part of understanding survival patterns over time (Ashauer et al. 
Table 2 Organism recovery times (95\% of recovery) based on different model types

\begin{tabular}{llll}
\hline Model & & Calibration data & $\begin{array}{l}95 \% \text { recovery } \\
\text { times (days) }\end{array}$ \\
\hline SD & \multirow{2}{*}{ Full } & Pulse toxicity & 1.5 \\
& & Acute toxicity & 2.3 \\
& & Both & 2.5 \\
& \multirow{3}{*}{ Reduced } & Pulse toxicity & 1.6 \\
& & Acute toxicity & 2.4 \\
& \multirow{3}{*}{ Foll } & Both & 2.8 \\
& & Pulse toxicity & 6.3 \\
& & Acute toxicity & 4.2 \\
& & Both & 4.2 \\
& Reduced & Pulse toxicity & 8.5 \\
& & Acute toxicity & 4.3 \\
& & Both & 4.8 \\
\hline
\end{tabular}

$S D$ Stochastic death model, IT Individual tolerance model, Full = Model including toxicokinetics, Reduced $=$ Model excluding toxicokinetics

2010; Butcher et al. 2006), which might suggest that TK may also be essential for predicting survival over time. Here we compared the goodness of fit between reduced and full models (Fig. 4). The difference in model structure between the full and reduced models is that the full model includes a pre-calibrated TK sub-model which simulates internal concentrations in the different exposure scenarios (Fig. 1). This is used as an input to the survival model, where it scales the organism damage. The reduced model skips the internal concentration and damage steps but instead, the internal concentration is scaled and can be described as a lumped variable for damage and internal concentration. In almost all cases, the reduced models resulted in the same or even higher log-likelihood than the full models. This can be explained by variation which the TK experiment brings to the survival model because the TK and TD experiments were not conducted simultaneously. One can conclude that in this example, measuring and simulating TK was not essential to achieve good predictions of survival. However, even if not necessary in survival models, TK provide important information on compound bioaccumulation potential and biotransformation, as well as mode of action, and therefore should not be disregarded.

TKTD models show potential to predict effects of multiple pulse exposure. The models have been calibrated using long term pulsed toxicity experiments (Ashauer et al. 2007a, b; Ashauer et al. 2010; Butcher et al. 2006; Meyer et al. 1995) or constant exposure experiments (Mancini 1983; Meyer et al. 1995). Using the toxicity data from constant exposures for model calibration would allow applying these models widely in risk assessment because this type of data has been and is generated in standard toxicity experiments. Here, we calibrated the TKTD models using pulsed toxicity data, acute toxicity data or both together. Therefore we could compare the goodness of fit and parameter estimates amongst calibration data sets. In addition, we were able to use parameters produced by either of the data sets to predict the effects in the other scenario and compare the simulation results with observations (model validation, see TRACE in Box SI-1). The calibration data had an influence on the parameter estimates (Table 1) and on the goodness of fit (Fig. 4). As expected, overall fit was the best if both data sets were used for model calibration, i.e. the total MPE was the lowest. In addition, the total likelihood (shown in Fig. 4 as combined likelihood value for acute and pulsed toxicity data above each model type) was maximised by fitting the models to both data sets together. When only one data set was used for the calibration, the choice of calibration data affected the predictive power of the model. If we compare the total likelihood values of the models calibrated with either pulsed toxicity or acute toxicity data, we see that the maximum likelihood is achieved using acute toxicity data for calibration. Similarly, previous studies have shown that the effects of time-varying exposure can be rather well predicted based on constant acute toxicity data (Mancini 1983; Meyer et al. 1995) but the effects of constant acute toxicity exposure are poorly predicted with time-varying toxicity data (Meyer et al. 1995). Meyer et al. (1995) stated that either the models did not mimic the processes well enough or there are different physiological processes which determine the toxicity under constant and time-varying exposure. Thus, the effects of time-varying exposure might be better predicted using data from time-varying exposure under different exposure regimes than using data from constant exposure studies (Meyer et al. 1995).

The choice of calibration data also had an effect on how the model predicts survival in a different exposure scenario. For example, the model calibrated using pulsed toxicity data mostly underestimated mortality in the acute toxicity scenario (Fig. 4). On the other hand, when acute toxicity data alone was used to calibrate the models, mortality in the pulsed exposure scenario was overestimated. In a risk assessment context, this overestimated mortality in the prediction could be acceptable. Thus one could calibrate TKTD models using already existing acute toxicity data because they provide protective predictions of survival in a pulsed (natural) exposure scenario. However, we here studied only one combination of chemical and organism, which is not sufficient to generalize this conclusion. More evidence is required before recommendations for appropriate model calibration data or model structures (e.g. with or without TK) can be made. Therefore studies on TKTD models and how they are able to predict 
time-varying exposure using acute or pulsed toxicity data should be conducted using more combinations of chemicals and species.

Acknowledgments This research has been financially supported by the European Union under the 7th Framework Programme (project acronym CREAM, contract number PITN-GA-2009-238148).

Conflict of interest The authors declare that they have no conflict of interest.

Open Access This article is distributed under the terms of the Creative Commons Attribution License which permits any use, distribution, and reproduction in any medium, provided the original author(s) and the source are credited.

\section{References}

Albert C, Ashauer R, Künsch HR, Reichert P (2012) Bayesian experimental design for a toxicokinetic-toxicodynamic model. J Stat Plan Inference 142:263-275

Anderson N (1979) Detritus processing by macroinvertebrates in stream ecosystems. Ann Rev Entomol 24:351-377

Ashauer R (2010) Toxicokinetic-toxicodynamic modelling in an individual based context: consequences of parameter variability. Ecol Model 221:1325-1328

Ashauer R, Brown CD (2008) Toxicodynamic assumptions in ecotoxicological hazard models. Environ Toxicol Chem 27: $1817-1821$

Ashauer R, Escher BI (2010) Advantages of toxicokinetic and toxicodynamic modelling in aquatic ecotoxicology and risk assessment. J Environ Monit 12:2056-2061

Ashauer R, Boxall A, Brown C (2006) Predicting effects on aquatic organisms from fluctuating or pulsed exposure to pesticides. Environ Toxicol Chem 25:1899-1912

Ashauer R, Boxall ABA, Brown CD (2007a) New ecotoxicological model to simulate survival of aquatic invertebrates after exposure to fluctuating and sequential pulses of pesticides. Environ Sci Technol 41:1480-1486

Ashauer R, Boxall ABA, Brown CD (2007b) Simulating toxicity of carbaryl to Gammarus pulex after sequential pulsed exposure. Environ Sci Technol 41:5528-5534

Ashauer R, Hintermeister A, Caravatti I, Kretschmann A, Escher BI (2010) Toxicokinetic and toxicodynamic modeling explains carry-over toxicity from exposure to diazinon by slow organism recovery. Environ Sci Technol 44:3963-3971

Ashauer R, Hintermeister A, O'Connor I, Elumelu M, Hollender J, Escher BI (2012) Significance of xenobiotic metabolism for bioaccumulation kinetics of organic chemicals in Gammarus pulex. Environ Sci Technol 46(6):3498-3508

Bedaux JJM, Kooijman SALM (1994) Statistical analysis of bioassays, based on hazard modelling. Environ Ecol Stat 1:303-314

Bliss CI (1935) The calculation of the dosage-mortality curve. Ann Appl Biol 22:134-167

Brock TCM, Alix A, Brown CD, Capri E, Gottesbüren BFF, Heimbach F, Lythgo CM, Schulz R, Streloke M (2010) Linking aquatic exposure and effects. SETAC, Pensacola, p 410

Butcher J, Diamond J, Bearr J, Latimer H, Klaine TS, Hoang T, Bowersox M (2006) Toxicity models of pulsed copper exposure to Pimephales promelas and Daphnia magna. Environ Toxicol Chem 25:2541-2550
Curtis LR, Seim WK, Chapman GA (1985) Toxicity of fenvalerate to developing steelhead trout following continuous or intermittent exposure. J Toxicol Environ Health 15:445-457

Dauterman WC (1994) Adaptation to toxicants. In: Hodson E, Levi P (eds) Introduction to biochemical toxicology. Apleton and Lange, Norwalk

FOCUS (2001). FOCUS surface water scenarios in the EU evaluation process under 91/414/EEC, EC Document Reference SANCO/ 4802/2001-rev.2. Report of the FOCUS Working Group on Surface Water Scenarios, Brussel, Belgium, p 245

Jager T (2011) Some good reasons to ban ECx and related concepts in ecotoxicology. Environ Sci Technol 45:8180-8181

Jager T, Albert C, Preuss TG, Ashauer R (2011) General unified threshold model of survival: a toxicokinetic-toxicodynamic framework for ecotoxicology. Environ Sci Technol 45:2529-2540

Kallander DB, Fisher SW, Lydy MJ (1997) Recovery following pulsed exposure to organophosphorus and carbamate insecticides in the midge, Chironomus riparius. Arch Environ Contam Toxicol 33:29-33

Kooijman SALM, Bedaux JJM (1996) Some statistical properties of estimates of no-effect concentrations. Water Res 30:1724-1728

Kretschmann A, Ashauer R, Preuss TG, Spaak P, Escher BI, Hollender J (2011) Toxicokinetic model describing bioconcentration and biotransformation of diazinon in Daphnia magna. Environ Sci Technol 45:4995-5002

Kreuger J (1998) Pesticides in stream water within an agricultural catchment in southern Sweden, 1990-1996. Sci Total Environ 216:227-251

Lafont R, Mathieu M (2007) Steroids in aquatic invertebrates. Ecotoxicology 16:109-130

Liu C, Gin KYH, Chang VWC, Goh BPL, Reinhard M (2011) Novel perspectives on the bioaccumulation of PFCs: the concentration dependency. Environ Sci Technol 45:9758-9764

MacNeil C, Dick JTA, Elwood RW (1997) The trophic ecology of freshwater Gammarus spp. (Crustacea, Amphipoda): problems and perspectives concerning the functional feeding group concept. Biol Rev 72:349-364

Maeder V, Escher BI, Scheringer M, Hungerbühler K (2004) Toxic ratio as an indicator of the intrinsic toxicity in the assessment of persistent, bioaccumulative, and toxic chemicals. Environ Sci Technol 38:3659-3666

Mancini JL (1983) A method for calculating effects, on aquatic organisms, of time varying concentrations. Water Res 17: $1355-1362$

McCahon CP, Pascoe D (1991) Brief-exposure of first and fourth instar Chironomus riparius larvae to equivalent assumed doses of cadmium: effects on adult emergence. Water Air Soil Pollut 60:395-403

McCarty LS, Mackay D (1993) Enhancing ecotoxicological modeling and assessment. Body residues and modes of toxic action. Environ Sci Technol 27:1718-1728

Meyer JS, Gulley DD, Goodrich MS, Szmania DC, Brooks AS (1995) Modeling toxicity due to intermittent exposure of rainbow trout and common shiners to monochloramine. Environ Toxicol Chem 14:165-175

Naylor C, Maltby L, Calow P (1989) Scope for growth in Gammarus pulex, a freshwater benthic detritivore. Hydrobiologia 188-189: $517-523$

Newman MC, McCloskey JT (2000) The individual tolerance concept is not the sole explanation for the probit dose-effect model. Environ Toxicol Chem 19:520-526

Nuutinen S, Landrum PF, Schuler LJ, Kukkonen JVK, Lydy MJ (2003) Toxicokinetics of organic contaminants in Hyalella azteca. Arch Environ Contam Toxicol 44:467-475

Parsons JT, Surgeoner GA (1991) Acute toxicities of permethrin, fenitrothion, carbaryl and carbofuran to mosquito larvae during 
single-or multiple-pulse exposures. Environ Toxicol Chem 10: $1229-1233$

Péry ARR, Bedaux JJM, Zonneveld C, Kooijman SALM (2001) Analysis of bioassays with time-varying concentrations. Water Res 35:3825-3832

Reinert KH, Giddings JM, Judd L (2002) Effects analysis of timevarying or repeated exposures in aquatic ecological risk assessment of agrochemicals. Environ Toxicol Chem 21:1977-1992

Schmolke A, Thorbek P, DeAngelis DL, Grimm V (2010) Ecological models supporting environmental decision making: a strategy for the future. Trends Ecol Evol 25:479-486

Schulz R, Liess M (2000) Toxicity of fenvalerate to caddisfly larvae: chronic effects of 1- vs 10-h pulse-exposure with constant doses. Chemosphere 41:1511-1517
Traas TP, van Leeuwen CJ (2007) Ecotoxicological effects. In: van Leeuwen CJ, Hermens JLM (eds) Risk assessment of chemicals: an introduction. Springer, Dordrecht, pp 281-356

Wittmer IK, Bader HP, Scheidegger R, Singer H, Lück A, Hanke I, Carlsson C, Stamm C (2010) Significance of urban and agricultural land use for biocide and pesticide dynamics in surface waters. Water Res 44:2850-2862

Zarn JA, Brüschweiler BJ, Schlatter JR (2002) Azole fungicides affect mammalian steroidogenesis by inhibiting sterol $14 \alpha$-demethylase and aromatase. Environ Health Perspect 111:255-261

Zhao Y, Newman MC (2007) The theory underlying dose-response models influences predictions for intermittent exposures. Environ Toxicol Chem 26:543-547 